\section{Geschichte der Urologie}

Urologe $2020 \cdot 59: 585-594$

https://doi.org/10.1007/s00120-020-01209-6

Online publiziert: 4. Mai 2020

(C) Der/die Autor(en) 2020

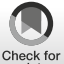

Friedrich H. Moll ${ }^{1,2,3}$. Marie-Isabelle Schwarzburger ${ }^{1,4}$

${ }^{1}$ Institut für Geschichte, Theorie und Ethik der Medizin, Heinrich-Heine-Universität, Düsseldorf, Deutschland

${ }^{2}$ Urologische Klinik, urologischer Arbeitsplatz Krankenhaus Merheim, Kliniken der Stadt Köln gGmbH, Köln, Deutschland

${ }^{3}$ Curator Museum, Bibliothek und Archiv zur Geschichte der Urologie, Deutsche Gesellschaft für Urologie e. V., Berlin-Düsseldorf, Berlin, Deutschland

${ }^{4}$ Institut für Geschichtswissenschaften I, Mittelalterliche Geschichte, Heinrich-Heine-Universität, Düsseldorf, Deutschland

\title{
St. Corona - eine Fürsprecherin gegen Seuchen?
}

\section{Eine Miszelle aus der Medizin- und Urologiegeschichte}

Franziska, um eine Schwangerschaft [11]. Ein weiterer ähnlicher Ritus ist für den Hl. Hypatius von Gangra ${ }^{1}$ dokumentiert [12].

Vom Mittelalter bis in die Neuzeit war es üblich, bei gesundheitlichen Beschwerden und/oder Einschränkungen bei den Heiligen der christlichen Kirche um Hilfe zu bitten, denn die Heiligen galten als direkte Verbindung zu Gott [13, 14]. Diese Fürbitten konnten durch ein Gebet, eine Opfergabe oder eine Wallfahrt geschehen, aber auch durch das Aufstellen eines Bildnisses und durch den Erwerb einer Reliquie. Hierbei ist es wichtig zu erwähnen, dass in einigen Fällen eine feste Absichtserklärung für eine Heilung schon reichte. Jeder Heilige war Patron für eine bestimmte Krankheit oder ein Leiden [15]. Darüber hinaus waren und sind Heilige Patrone von Städten und Berufsgruppen (so waren Caesarius von Nazianz, die Hl. Cosmas und Damian oder der Hl. Panthaleon Schutzheiligen der Ärzte). Bei widernatürlichen Gelüsten ("griechische Liebe“) wurde in Italien zum Beispiel der Hl. Bernadino oder der Hl. Isidor angerufen [16].

Das Wissen über Hagiotherapie, also die Anrufung von Schutzheiligen für

\footnotetext{
1 Eine Dokumentation zu urologischen Patrozinien in Düsseldorf und Umgebung ist in Vorbereitung.
}

das Kurieren einer Krankheit [17], wird heute kaum noch weitergegeben und einem breiten Publikum zur Verfügung gestellt. Zwar verfügt jede Kirche über Heiligenbildnisse und -altäre und damit die Möglichkeit, Fürbitte zu leisten, jedoch schwindet nach und nach die Bekanntheit der einzelnen Schutzpatrone. So ist das volkskundliche Wissen wie „Gebete bei Steinkolik“2 oder magische

\section{Infobox}

Andachtsbilder, Heiligenbilder, Fleißkärtchen mit religiösen Motiven wurden mit Entwicklung der Fotographie, der Chromolithographie im 19. Jahrhundert sowie des Mehrfarbenrasterdruckes im 20. Jahrhundert bei Benutzung von Schnellpresse zu Massenwaren. Als Wandbilddruck besonders mit "Schutzengelbildern" fanden religiösen Motive vermehrt Eingang in bürgerliche Wohnräume. In dieses Genre im Stil der Nazarener fielen auf nichtreligiöser Ebene der mythischen "Elfenreigen" oder der "Röhrende Hirsch", Motive, die viele Verlagshäuser im Programm hatten. Erst in den letzten Jahren wurde dieser Teil der Populärkultur wissenschaftliches Untersuchungsgebiet. Noch heute sind Angebote besonders in den romanischen Ländern und in Südamerika im Handel, meist mit religiösen Motiven.

2 „Liborius, halt für uns an, auf dass nicht Gries und Nierenstein die Strafen uns'rer Sünden sei'n." 


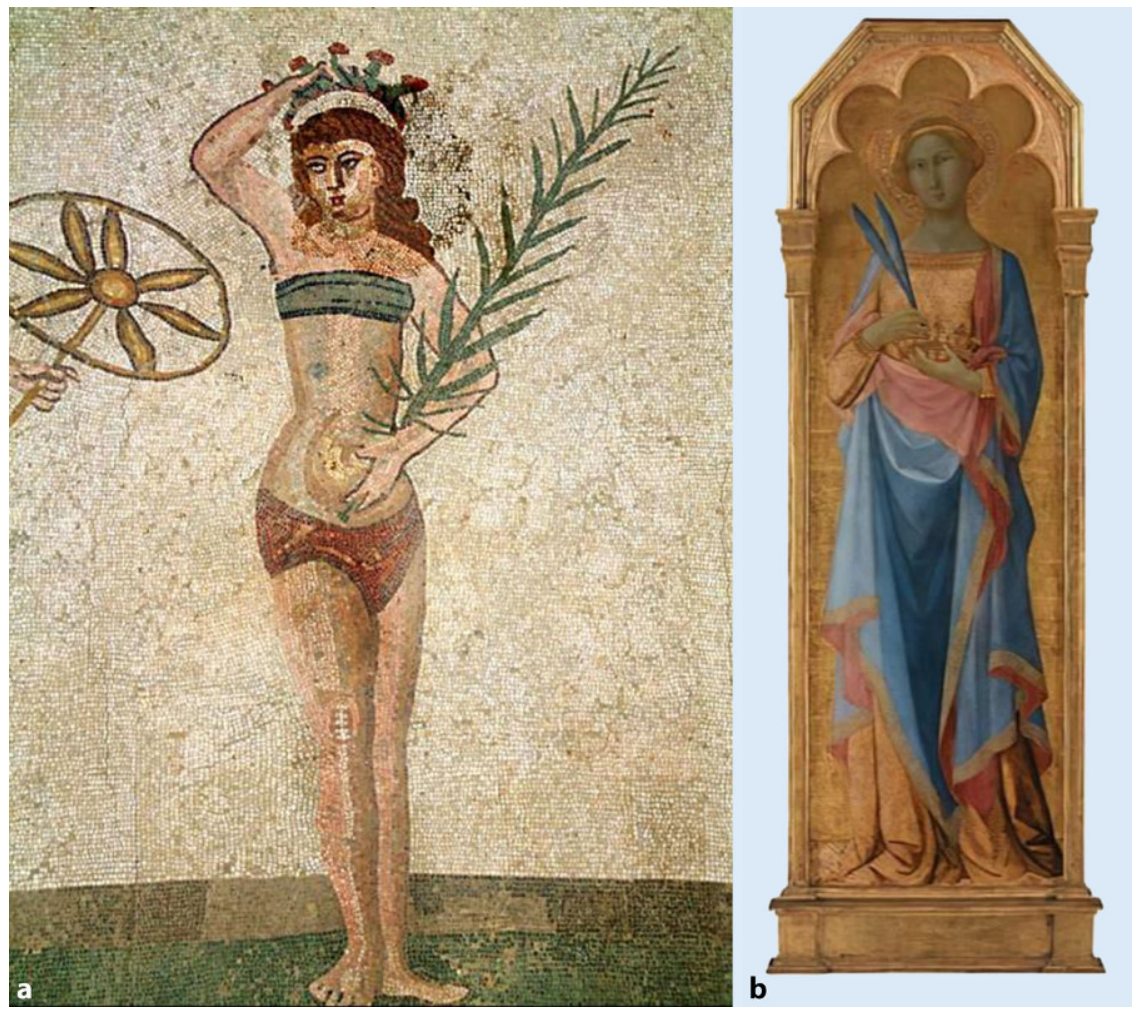

Abb. 1 A a Mosaikausschnitt "Mädchen im Bikini“ Villa Romana del Casale, Piazza Armerina, Provinz Enna, Sizilien. (Quelle: Wikipedia commons, als Vorbild der Darstellungsweise der "corona" als ein aus Blüten und Blättern geflochtene Kranz). b St. Corona als ikongraphisch ähnliche Darstellung mit Palme, um 1350 (Tempera auf Holz, Goldgrund), $155 \times 48 \mathrm{~cm}$, Meister der Palazzo Venezia Madonna (1340-1360), Teil des St. Viktor Altares, Siena, Statens Museum for Kunst, Copenhagen, Dänemark, Pendantbild zu einer Darstellung des HI. Viktor. (Mit freundl. Genehmigung, Repro Moll-Keyn ebenfalls wikipedia commons)

Handlungsanweisungen bei Steinen oder Pollutionen ${ }^{3,4,5}$ in der Regel verlorengegangen $[4,18,19]$.

\section{Heilige Corona (Stefan[i]a) - $S$ Corona matrona Martyr. in Aegyt.}

$\mathrm{Zu}$ Beginn des Jahres 2020 beherrscht das sog. "Coronavirus“ die Schlagzeilen, auch der urologischen Fachpresse [20]. Benannt ist diese Art von Virus nach dem

\footnotetext{
3 "Nieren-und Blasensteine treibt manab, wenn man Igelblut trocknet, pulverisiert und in Wasser eingibt." [18]

4 "Gegen den Stein im menschlichen Körper ist der Lapis nephriticus, in Silber oder Gold gefasst und am Halse getragen, ein treffliches Mittel, indem er den Stein im menschlichen Körper auflöst." [18]

${ }^{5}$ "Lasse den Samen von Sauerampfer durch einen unschuldigen Knaben sammeln und trage denselben in einem Beutelchen auf dem bloßen Leibe.. [18]
}

lateinischen Begriff corona „Krone“, da es eine Art Krone oder Strahlenkranz umgibt [21]. Das Covid-19-Virus gehört zu dieser Virusgruppe.

Die Hl. Corona verbindet mit dem zurzeit pandemischen Covid-19-Virus außer dem Namen noch mehr: Sie gilt in wenigen regionalen Bezügen als Schutzpatronin gegen Seuchen $[22]^{6}$, aber auch in Gelddingen (Österr. Münzeinheit bis 1924 „Krone“) und der Lotterie. Sie ist Patronin der Berufsgruppe der Metz-

\footnotetext{
${ }^{6}$ Nur in einer Quelle, die auf St. Corona am Wechsel zurückgeht, werden (Vieh-)Seuchen genannt. Wesentliche Lexika wie Schmidt und Beitl [23] geben Seuchen als Patrozinium nicht an. Hierauf weist Graf und Männig hin [24]. Seuchen waren seit alters her wichtige Gefahren in bäuerlichen Gemeinschaften. Es ist nachvollziehbar, dass man sich an eine örtlich besonders verehrte Heilige (in St. Corona am Wechsel ist Corona die Kirchen- und Ortspatronin) mit Gebeten in ganz besonderen Anliegen wandte. Nachweis nur Schäfer [25].
}

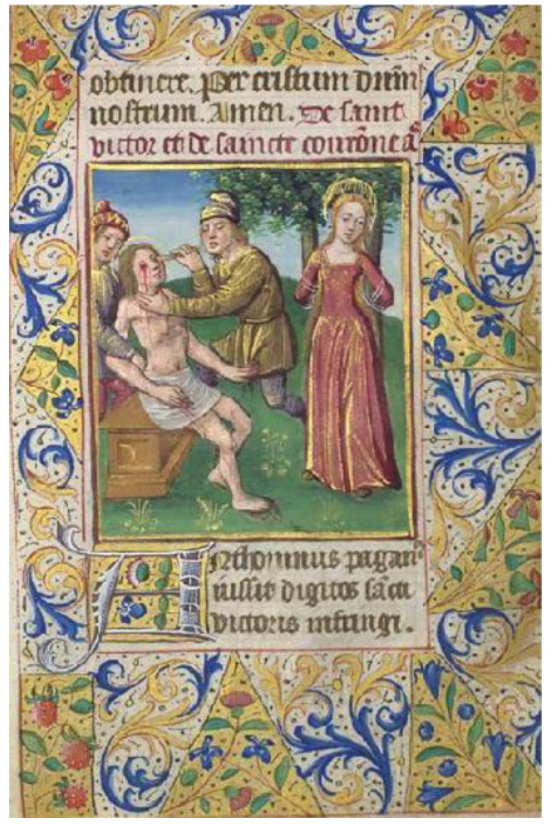

Abb. 2 \& Darstellung des Martyriums der HI. Corona und des HI. Viktor von Damaskus/Siena (nicht zu verwechseln mit dem HIViktor von Xanten) Miniatur aus einem französischen Stundenbuch ca. 1480. Man sieht symbolisch dieHI.Corona zwischen zwei Bäumen dargestellt, während das Martyrium des HI. Viktors - hier mit Ausstechen der Augen - plastisch hervorgehoben wird. (Quelle: https://commons.wikimedia.org/wiki/ File:SaintsVictor_and_Corona.JPG)

ger (Wortähnlichkeit caro lat: Fleisch). Als Reliquien verehrte Überreste von ihr finden sich in Aachen. Der mehr als 100 Jahre alte Schrein wird gerade restauriert und soll spätestens im Sommer in der Aachener Schatzkammer gezeigt werden [26]. Der katholische Gedenktag ist der 20. Februar/14. Mai, der orthodoxe der 11. November [25, 27].

Eine Krone war in der griechischen und römischen Antike ein zu kultischen Zwecken getragener Kranz aus Blumen, Blättern oder Zweigen bzw. die Nachbildung eines solchen Kranzes aus Metall. Demeter oder Kore werden so dargestellt. Der Palmzweig ist ein Friedenssymbol.

Auch der aus dem Griechischen $\sigma \tau \varepsilon \dot{\phi} \alpha v o \varsigma$ analog gebildete Name „Stephana" bedeutet Bekränzung, Kranz als Ehrenkranz, Kranz beim Opfer für die Götter und auch der Kranz, der im Kampf getragen wurde. Später wurde auch die Kopfbedeckung der Bischöfe 
der Ostkirche als Stephanos bzw. Mitra bezeichnet.

Laut Legende, die meistens auf die Acta Sanctorum [28] oder das Martyrologium Romanum [29] zurückgehen, soll Corona, in der Regel als Ehefrau des Hl. Viktor beschrieben, etwa 16 Jahre alt gewesen sein, als sie vor rund 1800 Jahren den frühchristlichen Märtyrertod um ca. 177? oder laut anderen Quellen um 303? starb, entweder unter Antonius Pius (Kaiser, 138-161) oder Gaius Aurelius Valerius Diocletianus, genannt Diokletian (Kaiser, 284-305). Ein römischer Statthalter habe die junge Christin mit Seilen zwischen zwei herabgebogene Palmen spannen lassen - durch das Zurückschnellen sei ihr Leib in Stücke gerissen worden. Diese Hinrichtungsart mit Zerren an den Gelenken und Abtrennung vom Körper war in Form der Vierteilung noch bis in das Mittelalter bekannt [30]. Es sind mehrere Orte des Martyriums von Damaskus, Antiochia (koptische Quellen), Alexandria bis Marseille bekannt [23, 28, 31-33].

In Syria sanctorum Martyrum Victoris et Coronae, sub Antonino Imperatore; ex quibus Victor a Sebastiano Judice variis et horrendis affectus est cruciatibus. Cum autem ipsum Corona, uxor cujusdam militis, coepisset beatum praedicare ob martyrii constantiam, vidit duas coronas de caelo lapsas, unam Victori et alteram sibi missam; cumque hoc audientibus cunctis testaretur, ipsa quidem inter arbores scissa, Victor vero decollatus est [29].

Der Name „Corona“, lateinisch „die Gekrönte“, weist ebenso wie der griechische Name „Stephana“ von Stephanus auf den allgemeinen Begriff „Märtyrerin“ hin.

Da beide Heilige durch ihre Legende eng miteinander verbunden sind, wird die Vita der Hl. Corona häufig mit der des Hl. Viktor, ihrem Ehemann (oder einem Kamerad ihres Mannes), zusammen dargestellt. Die Festtage gelten auch für beide Heilige. Eine Verehrung in Nordund Mittelitalien ist bereits für das 6. Jahrhundert belegt.

\section{Reliquien}

Reliquien (von lateinisch „reliquiae“ Zurückgelassenes, Überbleibsel) sind Gegenstände religiöser Verehrung. Besonders Körperteile oder Teile des persönlichen Besitzes eines Menschen, der von der katholischen Kirche als Heiliger verehrt wird, gehören hierzu [34]. Eine Reliquienverehrung ist Bestandteil katholischer Volksfrömmigkeit. In der evangelischen Kirche ist dieser Brauch unbekannt. Für den Reformator Martin Luther (1483-1546) waren Reliquien nicht heilig: „Es ist alles tot Ding, das niemand heiligen kann" [35]. Reliquien brachten im Mittelalter Macht, Ansehen, Pilger und Spenden. Das schuf eine gewisse Nachfrage nach ihnen. Und diese Nachfrage schuf wiederum ein Angebot [34].

Reliquien werden allgemein in drei Kategorien eingeteilt, die auch ihre Hierarchisierung wiederspiegeln. So sind unmittelbare Reliquien alle Körperteile der Heiligen. Dies können u. a. Knochen und Haare sein. (Reliquien erster Klasse). $\mathrm{Zu}$ den mittelbaren Reliquien oder echten Berührungsreliquien, gehören Gegenstände, die von den Heiligen noch zu ihren Lebzeiten berührt wurden. Die sog. „künstlichen“ sind jene, die echte Reliquien berührt haben [36].

Diese Kategorisierungen spiegeln nicht nur die Bedeutung der Reliquien selber, sondern auch die der besitzenden Institution wieder. So waren natürlich Reliquien der ersten Klasse neben den Christusreliquien besonders begehrt.

\section{Reliquientranslation}

Kaiser Otto III. (980-1002, Kaiser ab 996), der ebenfalls im Aachener Dom bestattet wurde, soll im Jahr 997 n. Chr. die Überreste der Hl. Corona und des Hl. Leopardus von Otricoli/Umbrien nach Aachen gebracht und in der Aachener Münsterkirche beigesetzt haben. Seither gelten beide Heilige als Mitpatrone des Aachener Marienstifts [37].

Eine andere Version lässt es möglich erscheinen, dass bereits Karl der Große (747/48-814, Kaiser ab 800) die Gebeine der Hl. Corona und des Hl. Leopardus nach Aachen überführen ließ.
Urologe 2020 $59: 585-594$

https://doi.org/10.1007/s00120-020-01209-6

(c) Der/die Autor(en) 2020

\section{F. H. Moll · M.-I. Schwarzburger}

\section{St. Corona - eine Fürsprecherin gegen Seuchen? Eine Miszelle aus der Medizin- und Urologiegeschichte}

\section{Zusammenfassung}

Hagiographie spielt in der Wissenschaftsgeschichte noch immer, gerade bei lokalgeschichtlichen Analysen, eine wichtige Rolle. Während sich in der Urologie das Wissen um den HI. Liborius gehalten hat, ist die Bekanntheit der Seuchenheiligen insbesondere denjenigen, die bei Geschlechtskrankheiten angerufen werden, in der Regel gesunken.

Schlüsselwörter

HI. Corona - Hagiographie in Medizin und Urologie · Erinnerungskultur · Volkskunde

\section{St. Corona-a patron saint against pandemics? A short essay on the history of medicine and urology}

\section{Abstract}

The knowledge of hagiography and hagiotherapy still plays an important role in the history of science, especially when focusing on specific aspects of history. While knowledge about St. Liborius persists in urology, knowledge about patron saints for pandemics, especially those who were called upon to treat venereal diseases, has diminished due to the association with nonappropriate sexual behavior.

\section{Keywords}

St. Corona $\cdot$ Hagiography in medicine and urology · Culture of remembrance · Folklore

Wann Corona in einem Kanonisationsprozess heiliggesprochen wurde, ist nicht bekannt. Es lassen sich aber durch Patrozinien Rückschlüsse ziehen. Da Leopardus und Corona zusammen als Conpatrone zur Weihe des Aachener Marienstiftes Ende des 8. Jahrhunderts benannt wurden, lässt darauf schließen, dass die Heiligsprechung vor dem Jahr 800 erfolgt sein muss. 

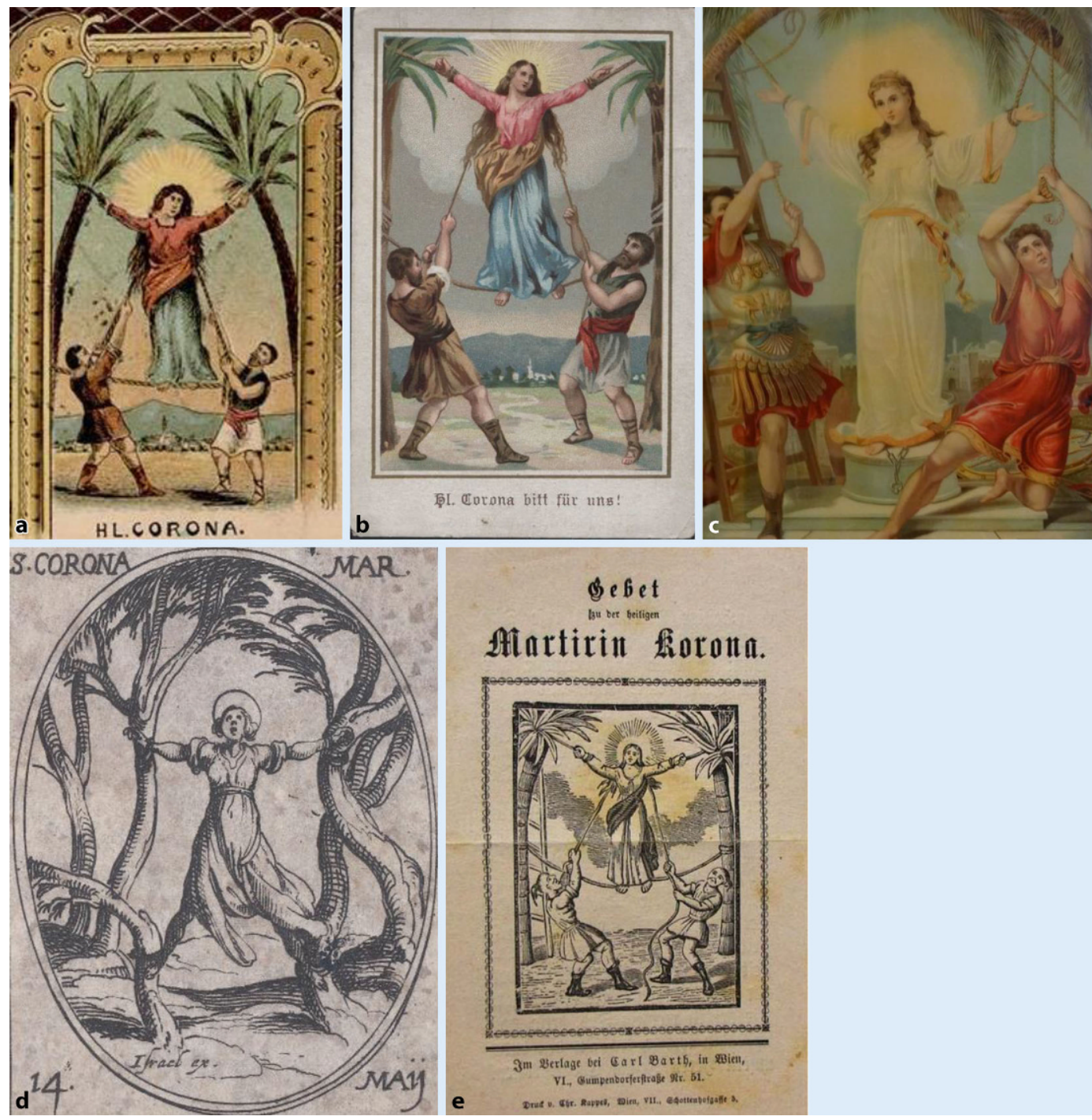

Abb. $3<$ a Postkartenausschnitt einer Karte aus St. Corona am Wechsel, NÖ mit der Marterdarstellung der $\mathrm{HI}$. Corona. b Ähnliches Bildmotiv eines Andachtsbildes, Verlag Carl Barth, Wien VI, um 1900. c Ähnliches Bildmotiv, ca. 1890. (Sammlung Moll Repro Moll-Keyn, mit freundl. Genehmigung). d Motiv von 1636 aus [24]; , „Les Images De Tous Les Saincts et Saintes de L'Année suivant le martyrologe Romain". (Sammlung Moll Repro Moll-Keyn, mit freundl. Genehmigung). e Einfache schwarzweiße Druckausführung des Marterbildes für ein Gebet $(10,5 \mathrm{~cm} \times 17 \mathrm{~cm})$. Verlag Carl Barth, Wien VI (Sammlung Moll Repro, mit freundl. Genehmigung)
Die Grabplatten sind bis heute im Aachener Dom zu sehen. 1843 - bei Grabungen auf der Suche nach dem Bestattungsort Karls des Großen [38] sowie 1910 wurden die Reliquiensärge aus Blei sowie die Gebeine ausgegraben und $a b$ 1911/1912 in Schreine gelegt $[39,40]$. Die Reliquiensärge werden in der Michaelskapelle des Aachener Doms aufbewahrt [41-44].

Kaiser Karl IV.(1346-1378, Regierung 1346-1378) brachte weitere Reliquien der Hl. Corona aus Feltre in den Prager Dom. Hierfür wird eine Rolle gespielt haben, dass man sich in Prag bemühte, Reliquien mit Namen von Heiligen, die Siegesoder Triumphnamen besaßen oder daran anklangen, zu erwerben [45].

Weiter befinden sich Reliquien seit 965 n. Chr. im Dom zu Bremen. Hier wird die
Hl. Corona viel verehrt, so finden sich Abbildungen von Ihr auf dem Chorgestühl des Bremer Doms und auf der Westempore [46]. In Bremen wurden auch Pilgerzeichen im Gitterguss mit Corona Motiv gefunden [47, 48].

Weitere Reliquien finden sich in Italien als frühen Ort der Verehrung beispielsweise in Castelfidardo bei Osimo an der Adriaküste bei Ancona oder in Otricoli/Umbrien s. oben (Santa Maria Assunta), wo auch der Hl. Leopardus verehrt wird $[49,50]$.

Dass es viele verschiedene Aufbewahrungsorte der Reliquien gibt, ist daher möglich, da oft schon kleinste Knochenfragmente in den Reliquiaren aufbewahrt werden. Weiter können einige auch aus der letzten Kategorie der Reliquien stam- men. Diese lässt die Anzahl an möglichen Reliquien exponentiell steigen.

\section{Patrozinien}

In Österreich sind Patrozinien in St. Corona am Wechsel (NÖ) sowie St. Corona am Schöpfl (Gemeinde Altenmarkt) bekannt. Ebenfalls gibt es ein Patrozinium St. Corona in Handlab bei Passau sowie in Arget, Gemeinde Sauerlach, Obb., sowie Unterzarnham (Kreis Mühldorf am Inn).

Im Bistum Regensburg sind sogar drei Patrozinien der heiligen Corona verzeichnet: die ehemalige Wallfahrtskirche St. Corona in Altenkirchen bei Frontenhausen, die Pfarrkirche St. Corona in Staudach bei Eggenfelden sowie die Nebenkirche Koppenwall in der Pfar- 


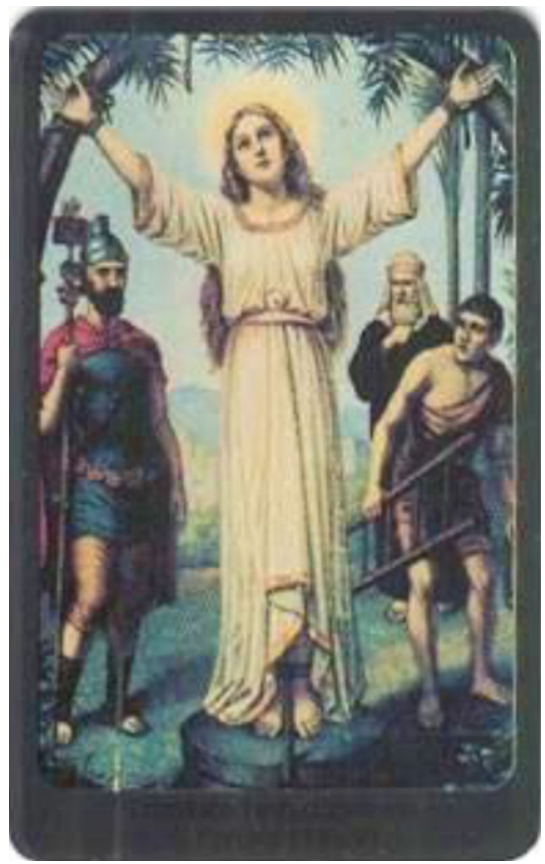

Abb. 4 A Fridolin Leiber (1843-1912) Heilige Corona, um $1900(50 \mathrm{~cm} \times 36 \mathrm{~cm})$. Chromolithographie, Sammlung Moll (Repro Moll-Keyn, mit freundl. Genehmigung)

rei Pfeffenhausen bei Landshut. Wahrscheinlich war der Corona-Kult über Böhmen und Niederösterreich auch ins südöstliche Bayern gekommen [51-53].

In Italien besteht ein Patrozinium in Vicenza, in Frankreich zusammen mit dem Hl. Viktor in Ennezat (Département Puy-de-Dôme) sowie in Italien in Grazzano Badoglio (Provinz Asti) und Feltre.

\section{Volkskundliche Aspekte des Corona-Kults}

Das Corona-Gebet (Kronengebet) ist ein volksmagisches Ritual, besonders in Niederösterreich und Böhmen, das während des 17. und 18. Jahrhunderts populär war. Es sollte zum Aufspüren verborgener Schätze dienen und wurde mehrfach erwähnt [54-56]. Gerichtsprozesse in der frühen Neuzeit, die sich mit magischer Schatzsuche befassten, siedelten das Delikt meist nicht im Bereich der Magie an, sondern werteten es als Betrug. Die Verbindung der Heiligenlegende hiermit blieb in der Forschung unklar, wahrscheinlich ist es auf die wortgleiche Münzbezeichnung „Krone“ zurückgeführt [57].
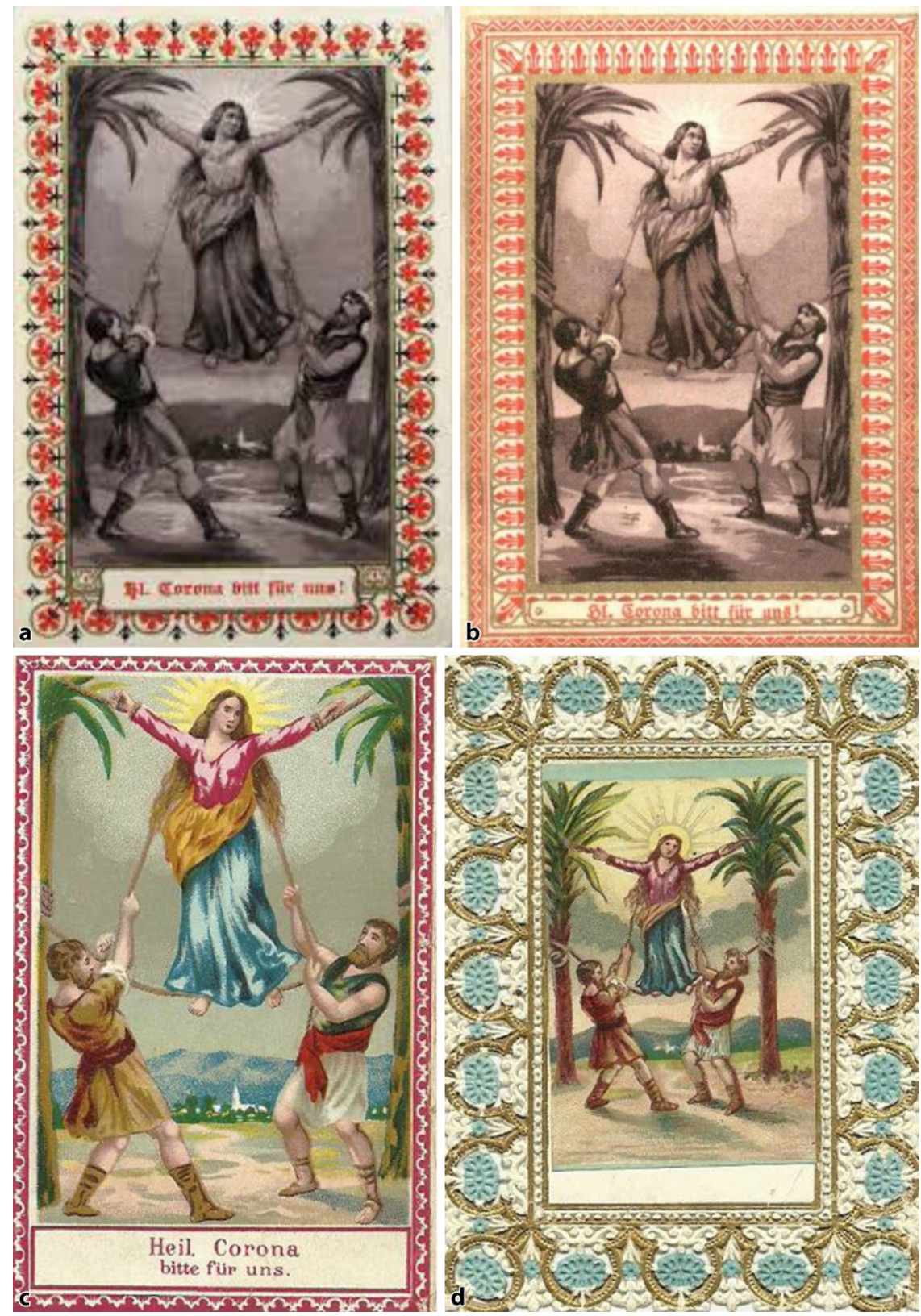

Abb. $5 \Delta$ Motivgleiche Abbildungen des Martyriums in Verschiedenen Farbgestaltungen und mit verschiedenen Umrandungen. Sammlung Moll (Repro Moll-Keyn, mit freundl. Genehmigung)

Gebete an die Hl. Corona lassen sich bis in das 20. Jahrhundert nachweisen $[58,59]$.

\section{Darstellungen der HI. Corona}

In der christlichen Kunst werden Heilige mit Attributen dargestellt, um diese kenntlich zu machen oder das Martyrium zu charakterisieren. Neben der Kleidung sind das oft Gegenstände oder beigeordnete Tiere [60,61].
Die Hl. Corona wird in der Kunst zumeist als junge Frau dargestellt, da sie mit 16 Jahren gestorben ist. Sie ist in den Darstellungen als Märtyrerin durch die Attribute Krone und Palmzweig zu erkennen oder anhand der Darstellung ihres Martyriums. Dieses zeigt sie, nach ihrer Legende, zwischen zwei über Kreuz gespannten Palmen. In vielen dieser Abbildung sind auch zwei römische Soldaten zu sehen, welche die Palmen gespannt halten. 


\section{Geschichte der Urologie}
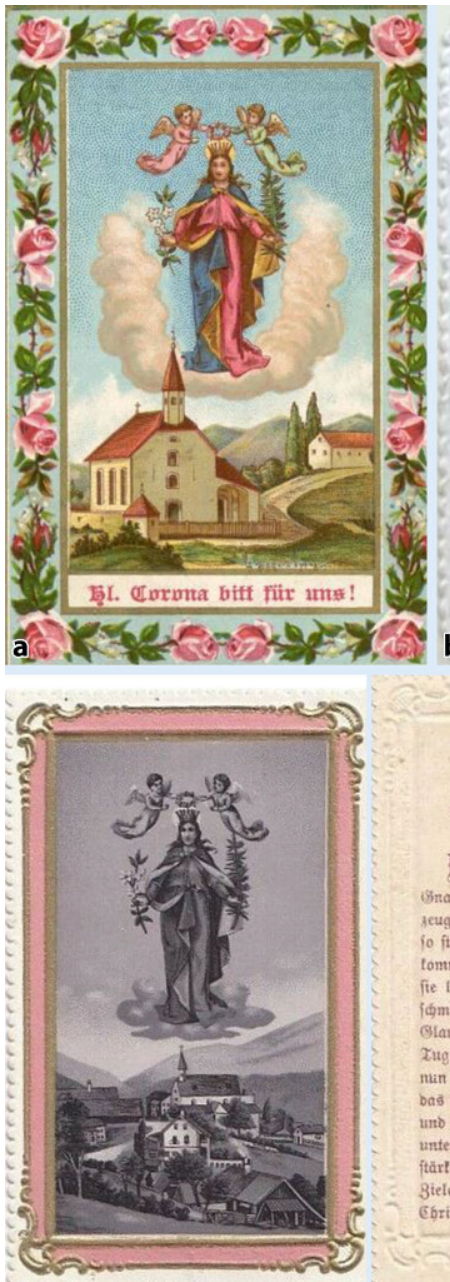

C1. Corona bei kirdberg am Wedsel.

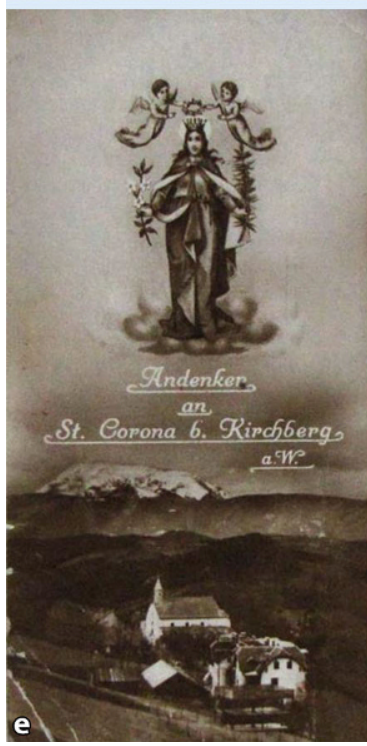

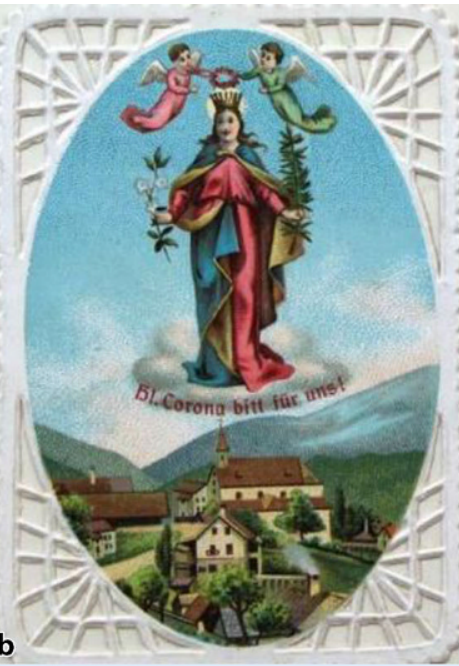

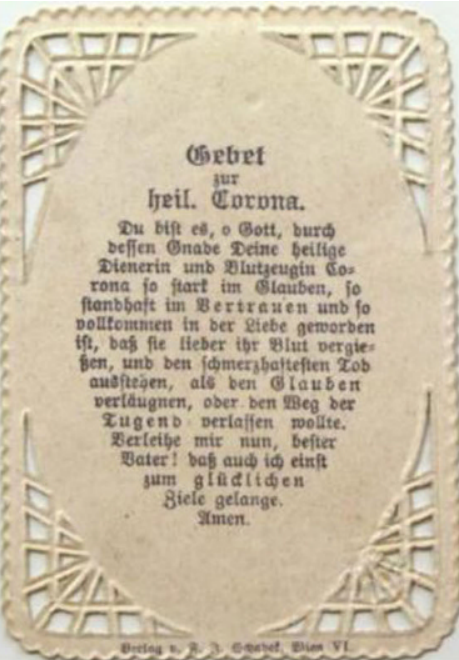

(5) ebef

jux britgen Giona.

㚜u bift cs, Bott, burch beffen Grabe Deine bet. Dienerin unt glut seugin Poroma io fart itm Grlaben. io ftantbhaft im teritauten unib fo volf tommen in ber giebs gemorben if , bas fie lieber ibe glit gerieten nub ben ímerglidften qut Dergiefent unb ben Ten Tumbert berläugnen, ober ben 28 eg ber Xugenb verlaffen wollte. Berleibe mis mint befter Bater! baf aud i由 burd bas Beciptel oifer Selitigen etmuntert, uno burd itre Fatrbitte in Slauben untertituly, in bet soffinung unb giebe fẫrter werbe unb einft gum glädlididen Biele gelange burc unferen serrm Sefu Gorititi. $\mathrm{gmen}$.

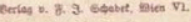
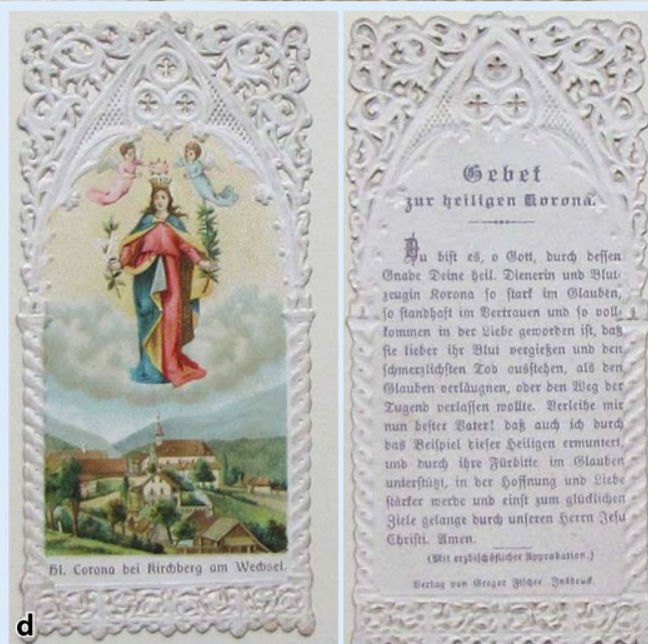

Dut bift esి, o Brott, Durd defilen (Bnabe Deinte beil. Dies nerin uno Blutzeugin Eorona fo ftark im Blauben, fo ftanto. haft im Bertrauen und fo voll fomment in ber \&iebe geworden ift, Dẩ fie lieber ibr 2 lut ver. giểeıt uno ben idjumerz̧lidjtten Tod ausfteben, als bett Blaus.

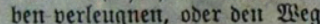
ber Tugend berlafient wollte. Berleibe mix nut befter $23 a t e r$. Dnß̧ aud) id) ourd) Das Beijpiel biejer \$eiligen ermuntert, uno Durd) ibre fö̈rbitte im (Slauben unterftitist, in Der ఏofinum!y uno qiebe ftärfer werbe uno eint sum glitufidjen Biele gelange burn unierent Serrn ieju Shrifti. 2Umett.

Frans Eqcum, giürnberg
Abb. $6<$ Motiv ähnliche Darstellungen der HI. Corona als Kirchen - Schutzpatronin, St. Corona am Wechsel N.Ö. Sammlung Moll (Repro Moll-Keyn, mit freundl.Genehmigung) 


\section{Darstellung als Märtyrerin}

Auf einem Altarbild wird die Hl. Corona nach römischen Vorbild (• Abb. 1a) mit Palmzweigen, die auf ihr Martyrium hindeuten und einer Krone dargestellt. Die kreisförmige Aureole ist nur schwer zu erkennen. Schon bei den Römern wurden Herrscher mit einem Nimbus (lat. Wolke) dargestellt [62].

\section{Darstellung des Martyriums (- Abb. 2)}

\section{Andachtsbilder mit dem Martyrium der HI. Corona als Massenware}

Die Andachtsbilder mit dem Martyrium der Hl. Corona (- Abb. 3a-c) sind von der Darstellung her zunächst sehr ähnlich. Abgebildet sind zwei römischen Soldaten, die die beiden Palmen gespannt halten, zwischen denen Corona gespannt wird. Auch hier ist sie eine junge Frau. Der Nimbus im Hintergrund erhebt sie, auch durch die fast schon schwebende Darstellung, in den Stand der Heiligen. Das Bildmotiv mit schwebender Corona ist schon von Jacques Collot (1592-1635) verwandt worden ([63]; • Abb. 3d) In der Abbildung aus einem Stundenbuch eines unbekannten Autors um 1480 (• Abb. 2) steht die Heilige noch zwischen Bäumen, die nicht als Palmen zu erkennen sind.

Das Bildmotiv der Marter eignet sich durch seine ausdrucksstarke Formensprache auch als schwarz-weiße Abbildung (• Abb. 3e).

Der Künstler Friedolin Leiber (18431912), der in Frankfurt für das Verlagshaus Edward Gustav May arbeitete und der für seine "Schutzengelbilder“ bekannt wurde, nutze ebenfalls das Motiv des Martyrium der Hl. Corona (• Abb. 4). Die Heilige wir von ihm mit einem weißem Kleid als Farbe der Unschuld und Reinheit dargestellt. Ansonsten überwiegen Darstellungen der Heiligen mit Kleidern in rot-blauer Farbe (Rot für Martyrium und Glaube, Blau für Himmel). Leibers Abbildung ist an die älteren Motive aus Österreich (St. Corona am Wechsel) deutlich angelehnt. Er lässt aber den Ortsbezug in der Landschaftsdarstellung im Hintergrund bewusst aus, wahrscheinlich, um die Verkaufsmöglichkeiten ohne einen expliziten lokalen Bezug zu steigern [64, 65]. Noch heute wird diese Abbildung über ein online Versandhaus vertrieben [66].

Die Corona-Andachtsabbildungen aus Österreich (• Abb. 5a-d sowie 6a-e) aus St. Corona am Wechsel wurden als Massenwaren an vielfältige Anforderungen des populären Geschmacks der Zeit und der Preisgestaltung angeglichen, nicht nur in der Farbgebung (- Abb. 5a-c). Bei einfarbigen Abbildungen wurde der Rand in besonderen Farben betont. Auch wurden verschiedene kurze Fürbitten oder Gebete eingedruckt. Die Blumenumrandungen waren teils nur aufgedruckt (• Abb. 5a-c) oder auch aufwendiger gestanzt bzw. geprägt und die Marterdarstellung als Chromolithographie eingeklebt (• Abb. 5e, f; [67, 68]).

\section{Darstellungen als Schutzpatronin einer Kirche}

In den folgenden Abbildungen ist die $\mathrm{Hl}$. Corona als Patronin der ihr geweihten Kirchen dargestellt. Anders als in den vorherigen Andachtsbildern, sieht man sie hier über der Kirche schwebend. Sie ist förmlich in den Himmel aufgestiegen, die zwei schwebenden Engel krönen sie mit einer Blätterkrone. Dieses Bildmotiv ist an zeitgenössische Mariendarstellungen unverkennbar angelehnt ([69]; - Abb. 6a-c).

Bei der Wallfahrtsandachtsabbildung (St. Corona am Wechsel) des Wiener Verlags Schabek $(5,4 \mathrm{~cm} \times 7,8 \mathrm{~cm}$; - Abb. 6b, c) lässt sich durch das aufgedruckte Gebet die Funktion der Abbildung als Andachtsbild gut erkennen, wie auch bei der des Innsbrucker Verlags Fischer $(5 \mathrm{~cm} \times 10,7 \mathrm{~cm}$; $-A b b .6 d)$ oder des Nürnberger Verlags Franz Schemm $(4,4 \mathrm{~cm} \times 8,8 \mathrm{~cm}$; $-A b b .6 e)$ bei motivgleichem Frontbild mit über der Kirche schwebenden $\mathrm{Hl}$. Corona.

\section{Fazit für die Praxis}

- Innerhalb der Medizin- und Urologiegeschichte hat sich in der Er- innerungskultur hagiographisches Wissen erhalten, das nicht nur in Bezeichnungen für Krankenhausstationen, die der Urologie gewidmet sind, seinen Ausdruck findet. Meist ist dies mit dem HI. Liborius als Patron gegen Harnsteine verbunden.

- Seuchenheilige sind innerhalb der Urologie bis auf den HI. Rochus zumeist in Vergessenheit geraten, insbesondere, da Syphilis und Geschlechtskrankheiten eher negativ konnotiert sind.

- Für die HI. Corona finden sich nur für den Ort St. Corona am Wechsel Hinweise für eine Verehrung bei Seuchen.

- Für die Urologie bleibt sie unbekannt.

\section{Korrespondenzadresse}

PD Dr. Friedrich H. Moll, M.A. FEBU

Institut für Geschichtswissenschaften I, Mittelalterliche Geschichte, Heinrich-HeineUniversität

Düsseldorf, Deutschland

friedrich.moll@uni-koeln.de

Funding. Open Access funding provided by Projekt DEAL.

\section{Einhaltung ethischer Richtlinien}

Interessenkonflikt. F.H. Moll und M.-I. Schwarzburger geben an, dass kein Interessenkonflikt besteht.

Für diesen Beitrag wurden von den Autoren keine Studien an Menschen oder Tieren durchgeführt. Für die aufgeführten Studien gelten die jeweils dort angegebenen ethischen Richtlinien.

Open Access. Dieser Artikel wird unter der Creative Commons Namensnennung 4.0 International Lizenz veröffentlicht, welche die Nutzung, Vervielfältigung, Bearbeitung, Verbreitung und Wiedergabe in jeglichem Medium und Format erlaubt, sofern Sie den/die ursprünglichen Autor(en) und die Quelle ordnungsgemäß nennen, einen Link zur Creative Commons Lizenz beifügen und angeben, ob Änderungen vorgenommen wurden.

Die in diesem Artikel enthaltenen Bilder und sonstiges Drittmaterial unterliegen ebenfalls der genannten Creative Commons Lizenz, sofern sich aus der Abbildungslegende nichts anderes ergibt. Sofern das betreffende Material nicht unter der genannten Creative Commons Lizenz steht und die betreffende Handlung nicht nach gesetzlichen Vorschriften erlaubt ist, ist für die oben aufgeführten Weiterverwendungen des Materials die Einwilligung des jeweiligen Rechteinhabers einzuholen. 
Weitere Details zur Lizenz entnehmen Sie bitte der Lizenzinformation auf http://creativecommons.org/ licenses/by/4.0/deed.de.

\section{Literatur}

1. Nahmer D (1994) Die lateinische Heiligenvita Eine Einführung in die lateinische Hagiographie. WBG, Darmstadt

2. Weitbrecht J, Benz M, Hammer A, Koch E, Nowakowski N, Seidel S, Traulsen J (2019) Legendarisches Erzählen. Schmidt, Berlin

3. Moll F (2014) Miszellen zur Urologie in der Kunst und Kulturgeschichte des Rheinlandes. In: Halling T, Moll F (Hrsg) Urologie im Rheinland. Springer, Berlin, S 144-155 https://doi.org/10. 1007/978-3-662-44698-0 8

4. Sparwasser H (1976) St. Liborius. Schutzpatron der Urologen? Urologe B 16:148-150

5. Döhlemann C, Ellert A, Durner J, Gockerell N, MeßmerE, VogeserM (2011) Infrarotspektroskopie von alten Harnsteinen aus den Votivgaben der Wallfahrtskirche Grafrath. Urologe 50:466-476. https://doi.org/10.1007/s00120-011-2505-y

6. Wisotzki P (1988) Das Benediktswunder Kaiser Heinrichs II. Entstehung und Entwicklung einer mittelalterlichen Heilungslegende. Medizinhist J 23:42-65

7. Schutzheilige Schutzpatrone Lexikon (2010) Schutzpatrone gegen Krankheit. http://www. kirchenweb.at/schutzpatrone/schutzheilige/ schutzpatrone_krankheit.htm. Zugegriffen: 3. Apr. 2020

8. Ökomenisches Heiligenlexikon (2020) Patronate: Krankheiten. https://www.heiligenlexikon.de/ Patronate/Patronate-Krankheiten.htm. Zugegriffen:3. Apr. 2020

9. Helios St. Elisabeth Klinik (2020) Station Rochus. https://www.helios-gesundheit.de/kliniken/ oberhausen/ihr-aufenthalt/unsere-stationen/ station-rochus/.Zugegriffen: 23. März 2020

10. Malteser Krankenhaus St. Hildegardis (2019) Station Liborius. https://www.malteser-krankenhauskoeln.de/uploads/media/Urologische_Klinik_ Lindenthal_WEB_15.pdf. Zugegriffen: 23. März 2020

11. Migg T (2018) Glaube macht schwanger: Santa Maria Francesca delle Cinque Piaghe. https://www. deutschlandfunk.de/kinderlosigkeit-glaubenmacht-schwanger.886.de.html?dram:article id=409475. Zugegriffen: 23 . März 2020

12. Fröhlich G, Schulz J (2015) Sant'Ippazio (St. Hypatius von Gangra). Ein süditalienischer Kult um Potenz und Pastinaken. Urologe 54:1806-1810. https://doi.org/10.1007/s00120-015-3969-y

13. Klaß G (1990) Hagiotherapie im Mittelalter, Analyse der Voraussetzungen und Elemente eines volksmedizinischen Heilverfahrens am Beispiel des Rheinlandes. Dissertation, Uni Bonn, S 91-93

14. Dross F, Ruisinger M (2009) Krisenzeiten: Pest, Lepra und ihre Patrone. Ruisinger M (Hrsg) Heilige und Heilkunst Katalog des Deutschen Medizinhistorischen Museums Ingolstadt. DMHM Ingolstadt, S23-38

15. Klaß G (1990) Hagiotherapie im Mittelalter, Analyse der Voraussetzungen und Elemente eines volksmedizinischen Heilverfahrens am Beispiel des Rheinlandes. Diss Uni Bonn $\mathrm{S} 93$

16. VoigtG(1881)DieWiederbelebung desklassischen Alterthums oder das erste Jahrhundert des Humanismus, 2. Aufl. Bd. II. Reimer, Berlin, S471-472
17. Klaß, G. (1990) Hagiotherapie im Mittelalter, Analyse der Voraussetzungen und Elemente eines volksmedizinischen Heilverfahrens am Beispiel des Rheinlandes. Diss Uni Bonn S 15

18. NN (2003) Sechstes u. siebentes Buch Mosis ode Der magisch-sympathetische Hausschatz, das ist Mosis magische Geisterkunst, das Geheimniß aller Geheimnisse, wortgetreu nach einer alten Handschriftmit staunenerregenden Abbildungen. Bohmeier, Leipzig, S 100 (Ohne Ort und ohne Jahr; Neudruck Berlin 1976; Nachdruck des Neudrucks)

19. Mursani D (1985) Der Heilige Liborius in Italien Urologe B 25:45-51

20. KriegmairMC, KowalewskiKF,Lange B, Heininger $A$, Speck T, Haas $H$ et al (2020) Urologie in der Corona-Virus-Pandemie - Leitfaden 4/20. Urologe 59:442-449. https://doi.org/10.1007/s00120020-01200-1

21. Bradburne A (1970) Antigenic relationships amongst Coronaviruses. Arch Gesamte Virusforsch 31:352-364 (bes. 352)

22. NN (1856) Die St. Corona=Capelle zu Kirchberg am Wechsel. Sonntagsbl Serverinus Ver Wien 1:788-790 (bes. 789)

23. Schmidt L, Beitl K (1994) Lemma Corona, hl. In: Kasper W (Hrsg) Lexikon für Theologie und Kirche, 3. Aufl. Bd. 2. Herder, Freiburg im Breisgau (Sp. 1315-1316)

24. Graf K (2020) St. Corona ist Seuchenpatronin, echt jetzt? https://archivalia.hypotheses.org/121643. Zugegriffen: 23. März2020

25. Schäfer J (2020) Corona (Stephana) Ökumenischen Heiligenlexikon. https://www.heiligenlexikon.de/ BiographienC/Corona_Stephana.html. Zugegriffen:23. März 2020

26. Reuters (2020) German cathedral dusts off relics of St Corona, patron of epidemics. https:// www.reuters.com/article/us-health-coronavirusgermany-saint-idUSKBN21C2PM. Zugegriffen: 3. Apr. 2020

27. Urban H (2017) Lexikon der Heiligen und Namenstage, 3. Aufl. Herder, Freiburg (2010 1. Aufl.)

28. Henschen G, Paperbrochio D (1866) Acta Sanctorum, Maii Tomus Tertius Palm Paris, S267-271

29. introibo.net (2015) Martyrologium romanum Gregorii XIII., jussu editum Urbani VIII. et Clementis $X$. auctoritate recognitum ac deinde anno MDCCXLIX. Benedicti XIV. labore et studio auctum et castigatum. (S 198). https://introibo.net/ download/brevier/martyrologium_latein.pdf. Zugegriffen:23. März 2020

30. QuanterR (1901) Die Leibes-und Lebensstrafen bei allen Völkern und zu allen Zeiten. Leipziger Verlag, Leipzig (Nachdr. 1970Scientia, Aalen)

31. Torsy J, Kracht HJ (2008) Der große Namenstagskalender, 12. Aufl. Herder, Freiburg

32. Stadler JE, Heim FJ, Ginal J (1882) SS Viktor et Corona. Vollständiges Heiligen-Lexikon oder Lebensgeschichten aller Heiligen, Seligen etc. aller Orte und aller Jahrhunderte, deren Andenken in der katholischen Kirche gefeiert oder sonst geehrt wird Bd. 5. B. Schmid'sche Verlagsbuchhandlung, Augsburg, S679 (unter Bezugnahme auf das damit in Verbindung stehende Kritische, Alterthümliche, Liturgische und Symbolische, in alphabetischer Ordnung)

33. PreguntaSantoral.es (2013) Santos Estefanía (Corona) y Víctor, mártires. http://www. preguntasantoral.es/2013/09/santos-victor-ycorona/.Zugegriffen: 23. März2020

34. Angenendt A (1997) Heilige und Reliquien: die Geschichte ihres Kultes vom frühen Christentum bis zur Gegenwart. CHBeck, München
35. Luther M (1910) Der große Katechismus, das dritte Gebet. In: Knaake JKF et al (Hrsg) Katechismuspredigten 1528; Großer und Kleiner Katechismus 1529, WA 301. Kritische Gesamtausgabe D. Martin Luthers Werke, Bd. 30/1. Böhlau, Weimar, S 145

36. Klaß, G. (1990) Hagiotherapie im Mittelalter, Analyse der Voraussetzungen und Elemente eines volksmedizinischen Heilverfahrens am Beispiel des Rheinlandes. Diss. Uni Bonn S 107-109.

37. Teichmann E (1925) Über die Heiligen. Märtyrer Leopardus und Corona im Aachener Münster. ZAachen Geschichtsver 51:374-381 (insb. 374)

38. Käntzeler PS (1863) Die neuesten Nachgrabungen in der Aachener Münsterkirche zur Auffindung der Gruft Karls d. Gr. Bonn Jahrb 33/34:206-223 (insb. 215)

39. Toben H (2020) Reliquien von St. Corona liegen in Aachen: Die Gekrönte. https://www.domradio. de/themen/corona/2020-03-20/die-gekroentereliquien-von-st-corona-liegen-aachen. Zugegriffen:23. Apr. 2020

40. Döring 0 (1913) Ein Meisterwerk kirchlicher Goldschmiedekunst. Christl Kunst 10:9-14

41. Teichmann E (1929) Über die Heiligen Märtyrer Leopardus und Corona im Aachener Münster. ZAachen Geschichtsver 51:374-381 (insb. 374)

42. Giersiepen H (1991) Zwei Reliquiensärge aus Blei im Aachener Dom. Z Aachen Geschichtsver 97:335-349 (insb.337)

43. Giersiepen H (1992) Die Inschriften des Aachener Doms. Reichert, Wiesbaden, S14

44. Giersiepen H (1992) Deutsche Inschriften Online DI 31 Dom Aachen, Nr 16 Michaelskapelle 1992 DI 31. http://www.inschriften.net/aachen-dom/ inschrift/nr/di031-0016.html\#content. Zugegriffen: 23. März 2020

45. Machilek F (1985) Reliquientransfer und Politik. ZurVerehrung des hl. Burgunderkönigs Sigismund (um 474-524), unter besonderer Berücksichtigung Polens, Böhmens und Ungarns im 14. und 15. Jahrhundert Reliquientransfer und Politik. Brücken Ger Jahrb 1:59-98 (insb. 67)

46. Löhr A (1988) Die Heilige Corona und ihre mittelalterlichen Darstellungen in Bremen, Festschrift für Wilhelm Lührs und Klaus Schwarz. Brem Jahrb 88:47-58

47. Pilgerzeichendatenbank (2020) Detailansicht von Pilgerzeichen \#8. http://www.pilgerzeichen.de/ item/pz/8.Zugegriffen:23. März 2020

48. Wittstock J (1998) Der Bremer Pilgerzeichen-Fund. Jakobus Stud 9:85-107 (bes. 104)

49. Jacobilli L (2008) Sala Bolognese: SS Victor and Corona. In: Vite de' Santi e Beati dell' Umbria, Bd. I, S494-501

50. Stefan A (2013) Saint Corona-the First Patron Saint of Medieval Brasov? Stud Hist 58(1):201-226

51. Spirkner B (1938) Zum Corona-Kult, Bauernheilige und Patronin der Schatz-Gräber. Volk Volkstum 3:300-313

52. Schmidt L (1951) Zur Verehrung der hl. Corona in Bayern und Österreich. Bayr Jahrb Volkskd 2:69-79

53. Bauer A (1956) Zur Verehrung der HI. Corona in Oberbayern. Bayr Jahrb Volkskd 5:64-69

54. Priesner C (2011) Der zu vielen Wissenschaften anweisende curiöse Künstler. Alchemie, Volksmagie und Volksmedizin in barocken Hausbüchern. Sudhoffs Arch 95:170-208

55. Jakoby A (1987) Coronagebet. In: Stäubli HB, Hoffmann-Kreyer E (Hrsg) Handwörterbuch des deutschen Aberglaubens, Bd. 2. de Gruyter, Berlin, S106-107

56. Dillinger J (2007) Hexen und Magie. Campus, Frankfurt/New York, S37 
57. Wolf HM (2020) Corona, hl. https://austriaforum.org/af/Wissenssammlungen/ABC_zur Volkskunde_\%C3\%96sterreichs/Corona\%2C_hl. Zugegriffen: 31. März 2020

58. Universitätsbibliothek der Humboldt-Universität zu Berlin (2020) XV. Der Heiligen Corona Schatzgebet (1636), Handschriftliche Schätze aus Kloster=Bibliotheken umfassend sämmtliche vierzig Hauptwerke über Magie, verborgene Kräfte, Offenbarungen geheimste Wissenschaften, (1734-1810). https://www.digi-hub.de/viewer/ image/BV043193254/5/. Zugegriffen: 23. März 2020

59. Staatliche Museen zu Berlin - Preußischer Kulturbesitz (2020) Gebete an die heilige Corona Ertzschatzmeisterin über die verborgenen Schätze, Vorsprecherin der armen Leute und Gebieterin der bösen Geister (1908?). http://www.smb-digital. de/eMuseumPlus?service $=$ Externallnterface $\&$ module $=$ collection\&objectld $=2398655$. Zugegrif fen:23. März 2020

60. Alt H (1845) Die Heiligenbilder oder die bildende Kunst und die theologische Wissenschaft in ihrem gegenseitigem Verständnis historisch dargestellt. Plahn, Berlin
61. Detzel H (1896) Christlichen Ikonographie. Ein Handbuch zum Verständnis der christlichen Kunst. Die bildliche Darstellung der Heiligen Bd.2. Herder Freiburg, $\mathrm{S} 668$

62. Warland R(2013) Nimbus. In: Reallexikon für Antike und Christentum, Bd. 25. Hiersemann, Stuttgart, S915-938

63. Callot J (1636) Les Images De Tous Les Saincts et Saintes de L'Année suivant le martyrologe Romain... dédiées à Monseigneur l'Eminentissime Cardinal de Richelieu. Israeil Henriet, Paris

64. Thomas-Ziegler S (1992) Röhrender Hirsch und Betende Hände. Bildmotive und Funktion des populären Wandschmucks Bd. 49. Habelt, Köln, Bonn

65. Brückner W (2000) Gesammelte Schriften. Kleinbürgerlicher und wohlstandsbürgerlicher Wandschmuck im 20. Jahrhundert. Kunst und Konsum - Massenbilderforschung. Volkskunde als historische Kulturwissenschaft 6, Veröffentlichungen zu Volkskunde und Kulturgeschichte, Bd. 82. Verlag der Bayrischen Blätter für Volkskunde, Würzburg S407-444

66. Google (2020) Bildersuche Heilige

Corona. https://www.google.de/

search?q=sHeilige + Corona\&tbm $=i s c h \&$
ved=2ahUKEwj7y9b04rXoAhUP4oUKHc4CAdEO2 cCegQ|ABAA\&oq=sHeilige+Corona\&gs_l=img. 3...5105.8022..8119...0.0..0.269.2535.0j11j4...... 0....1..gws-wiz-img.......0i67j0j0i19.9_f9743eQsA\& ei $=2$ WF7Xrv7MI_ElwTOhYSIDQ\&bih=910\& biw $=1860 \#$ imgrc $=$ FqSpDUPDyFA10M $\&$ imgdii=dBuoL2pTd7TA0M. Zugegriffen: 23. März 2020

67. Beer M, Rehm U (2004) Das kleine Andachtsbild, Graphik vom 16. zum 20. Jahrhundert. Auswahlkatalog, Museum Schnütgen Köln. Olms, Hildesheim

68. Schade K (1996) Andachtsbild: die Geschichte eines kunsthistorischen Begriffs. Verlag und Datenbankfür Geisteswissenschaften, Weimar

69. Mendelsohn H (2012) Die Engel in der bildenden Kunst. Ein Beitrag zur Kunstgeschichte der Gotik und in der Renaissance. Unikum, Barsinghausen, S50-55

\section{e.Medpedia: Die neue Online-Enzyklopädie für Ärzte}

e.Medpedia ist die neue digitale Enzyklopädie für Ärzte und ermöglicht das schnelle Nachschlagen auf Basis medizinischer Standardwerke von Springer. Die über Peer-ReviewVerfahren begutachteten Einträge werden von über 2.800 erfahrenen klinischen Experten verfasst und fortlaufend aktualisiert.

- e.Medpedia enthält alle Inhalte von über 20 etablierten Referenzwerken von Springer

- Inklusive unzähliger Abbildungen, klinischer Bilder, Tabellen und Schemata sowie Videos

- Verfasst von über 2.800 renommierten Fachärzten, gesichert durch Peer Review-Verfahren

- Komfortable Suchfunktion mit schneller Erkennung der Suchwörter

- Über 7.000 Querverlinkungen zwischen den einzelnen Einträgen

- Die bestehenden Einträge werden fortlaufend aktualisiert

-Weitere Fachgebiete werden kontinuierlich erweitert

- Mobile Nutzung über Smartphones - online und offline mit der e.Medpedia App für iOS- und Android-Geräte

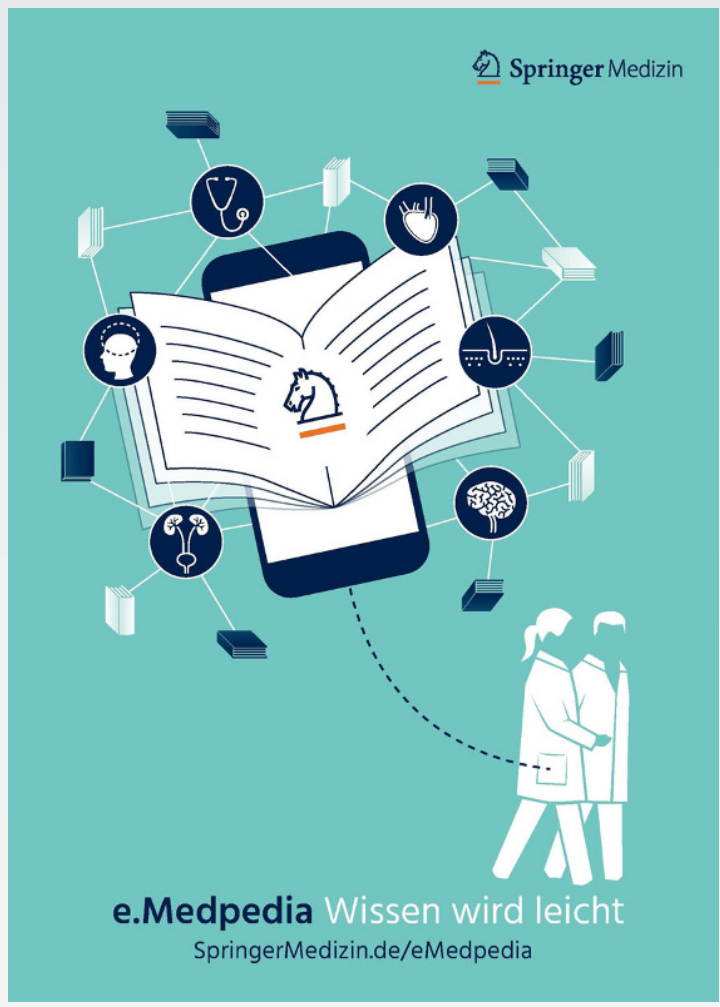


Hier steht eine Anzeige.

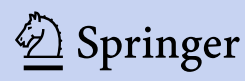

